Mass Profiles and Shapes of Cosmological Structures

G. Mamon, F. Combes, C. Deffayet, B. Fort (eds)

EAS Publications Series, Vol. ?, 2005

\title{
CONTRACTION OF DARK MATTER HALOS IN RESPONSE TO CONDENSATION OF BARYONS
}

\author{
Oleg Y. Gnedin ${ }^{1}$
}

\begin{abstract}
The cooling of baryons in the centers of dark matter halos leads to a more concentrated dark matter distribution. This effect has traditionally been calculated using the model of adiabatic contraction, which assumes spherical symmetry, while in hierarchical formation scenarios halos grow via multiple violent mergers. We test the adiabatic contraction model in high-resolution cosmological simulations and find that the dissipation of gas indeed increases the density of dark matter and steepens its radial profile compared to the case without cooling. Although the standard model systematically overpredicts the increase of dark matter density, a simple modification of the assumed invariant from $M(r) r$ to $M(\bar{r}) r$, where $\bar{r}$ is the orbit-averaged particle position, reproduces the simulated profiles within $10 \%$.
\end{abstract}

\section{Testing the Adiabatic Contraction Model}

In hierarchical galaxy formation models, stars are formed in condensations of cooled baryons within dark matter halos. The response of dark matter to baryonic infall has traditionally been calculated using the model of adiabatic contraction (AC). The standard model of AC, introduced by Blumenthal et al. (1986), assumes spherical symmetry, homologous contraction, circular particle orbits, and conservation of the angular momentum: $M(r) r=$ const, where $M(r)$ is the total mass enclosed within radius $r$. Because of the simplicity of this model, it has been routinely applied in the modeling of galaxies and clusters of galaxies.

It is amazing, however, that the model of AC has not been tested in a cosmological context. The hierarchical formation scenario is considerably more complex than the simple picture of quiescent cooling in a static spherical halo. Each halo is assembled via a series of mergers of smaller halos, in which the cooling of gas and contraction of dark matter occur separately. The gas can be re-heated by shocks

\footnotetext{
${ }^{1}$ Ohio State University, Department of Astronomy; ognedin@astronomy.ohio-state.edu
} 


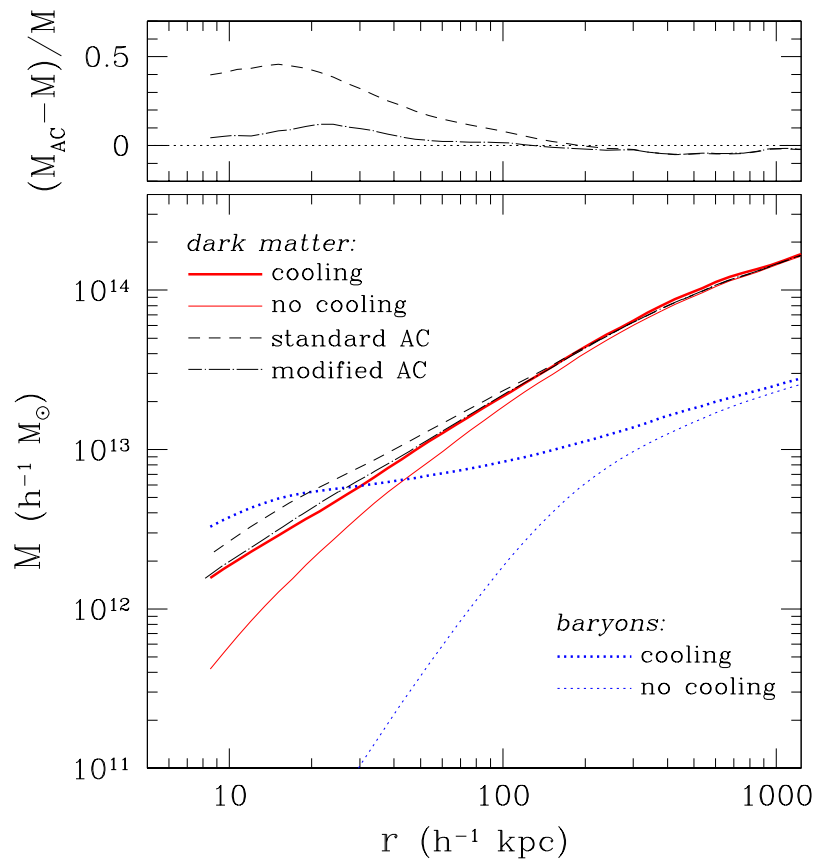

Fig. 1. Enclosed mass profiles of dark matter (solid) and baryons (gas+stars, dotted) in the adiabatic (thin) and cooling (thick) runs in a simulated cluster of galaxies. Dashed curve shows the prediction of the standard AC model, while dot-dashed curve shows the improved model. The profiles are truncated at four resolution elements of the simulation. Top panel: relative mass difference between the dark matter profile in the cooling run and the prediction of the standard AC model (dashed) or our modified model (dot-dashed).

and star formation feedback, and in addition early-type galaxies may undergo dissipationless mergers after the gas is exhausted.

In order to test the AC model, we have analyzed high-resolution cosmological simulations of eight cluster-sized and one galaxy-sized systems in a concordance $\Lambda$ CDM model (Gnedin et al. 2004). The simulations are performed with the Adaptive Refinement Tree (ART) $N$-body+gasdynamics code (Kravtsov 1999). For each halo, we analyze two sets of simulations which start from the same initial conditions but include different physical processes. The first set of simulations follows the dynamics of gas "adiabatically", i.e. without radiative cooling. The second set of simulations includes gas cooling and heating, and star formation. The difference in the halo profiles between the two runs is therefore due to the condensation of baryons.

Figures 19and2 show that cooling leads to an increase in the dark matter density in a representative cluster of galaxies within $r<50 h^{-1} \mathrm{kpc}$ or $r / r_{\mathrm{vir}}<0.04$. The difference in the enclosed mass increases substantially with decreasing radius. Note 


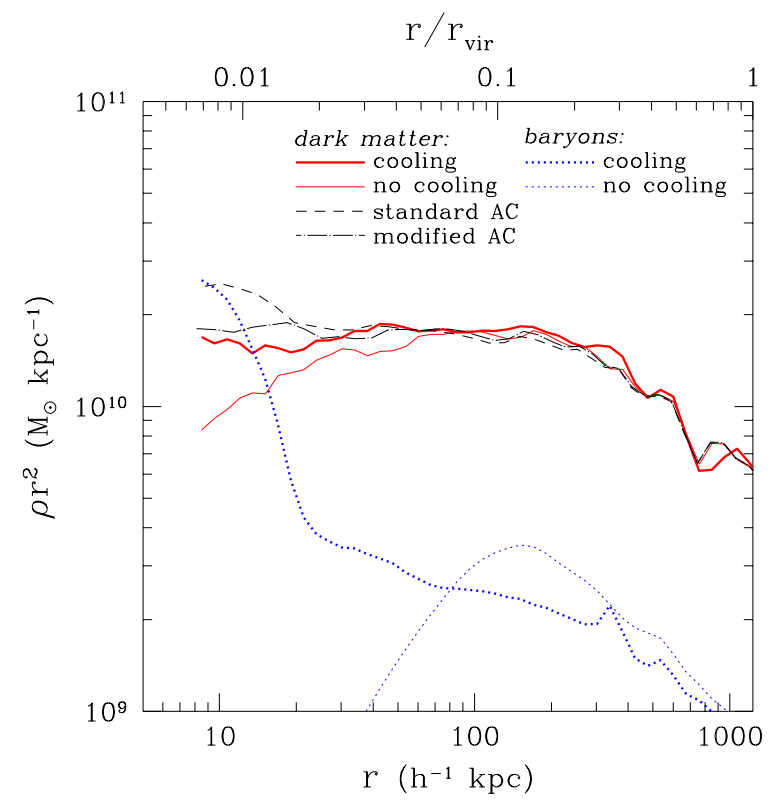

Fig. 2. Density profile of the cluster shown in Fig. 1 with the same line types. We plot the combination $\rho(r) r^{2}$ in order to emphasize the differences at small radii.

that although the simulations may suffer from the "overcooling of baryons", we can still test whether the AC model works. In fact, the larger effect of cooling allows us to emphasize the difference between the simulations and the model. The standard model of AC predicts the overall mass enhancement but systematically overestimates its magnitude in the inner regions. This effect has already been noticed in the original study of Blumenthal et al. (1986).

In the galaxy-sized halo at $z=4$ (Fig. 3) the dark matter density is enhanced within a larger fraction of the virial radius, $r / r_{\mathrm{vir}}<0.1$, than in the cluster runs. It is due to a considerably higher fraction of cold gas in the galaxy run $(80 \%$ vs. $20-30 \%$ for clusters), as well as a different mix of gas and stars (most of the baryon mass within the central regions of clusters is in the stellar component of the $\mathrm{cD}$ galaxy, while the galaxy at $z=4$ is very gas-rich). Because of the differences in the gas densities, temperatures, and cooling times, the cluster and galaxy simulations probe qualitatively different regimes of central baryon condensation. In both regimes the AC model works surprisingly well.

Nevertheless, many of the assumptions of the standard AC model are not valid. The orbits of particles in realistic dark matter halos are highly eccentric. For such orbits the combination $M(r) r$, which forms the basis of the standard AC model, varies with the orbital phase and is not adiabatically invariant. A better version of AC model can be constructed by taking into account eccentricities of particle orbits. An exact analytic form of the adiabatic invariant is not known in general 


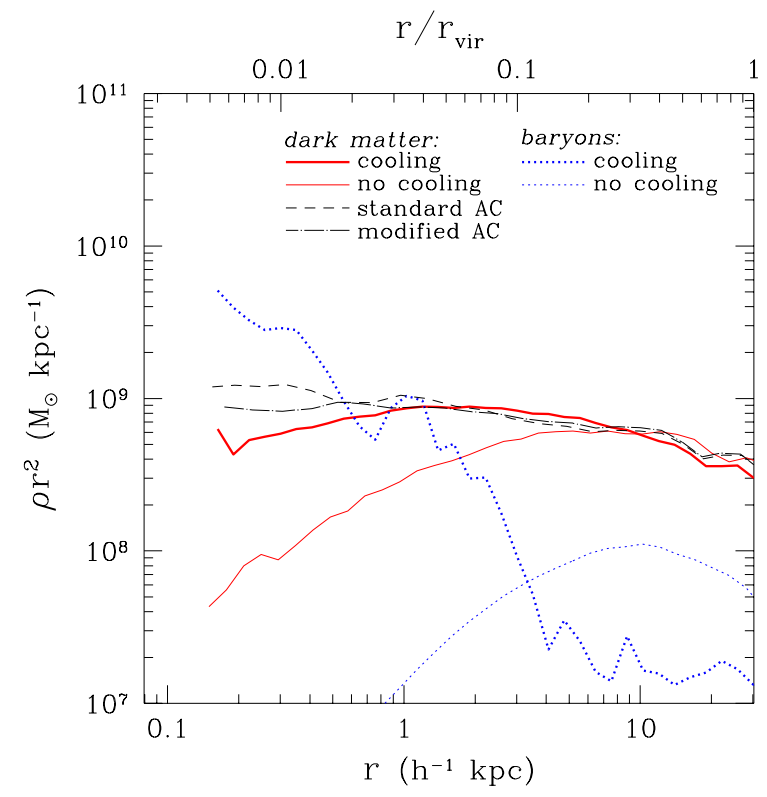

Fig. 3. Density profile in the galaxy-sized halo at $z=4$. Lines types are as in Fig. 1]

case, but Sellwood \& McGaugh (2005) suggest a numerical solution based on remapping the distribution function. However, this exact solution to the spherical collapse problem with conserved orbital actions may not necessarily apply in the case of hierarchical merging, where the action variables do change. Instead, we can look for a convenient approximation to the realistic evolution of halos in the cosmological simulations.

We proposed a modified adiabatic contraction model (Gnedin et al. 2004) based on conservation of the product of the current radius of a spherical shell, $r$, and the mass enclosed within the orbit-averaged radius, $\bar{r}$ :

$$
M(\bar{r}) r=\text { const. }
$$

The distribution of particle orbits with the energies and angular momenta found in cosmological simulations is close to isotropic, with a wide range of eccentricities. We found that in the range $10^{-3}<r / r_{\text {vir }}<1$, the orbit-averaged radius for particles at a current radius $r$ can be described by a power law relation

$$
\bar{x}=A x^{w}, \quad x \equiv r / r_{\mathrm{vir}},
$$

with small variations in the parameters $A \approx 0.85 \pm 0.05$ and $w \approx 0.8 \pm 0.02$ from halo to halo and from epoch to epoch. These two equations allow one to calculate the final profile of a hierarchically-assembled dark matter halo.

Figures 1 3 show that the modified model provides a more accurate description of the simulation results than the standard model. Although there are still differ- 
ences between the model and simulation profiles, the model does not systematic over- or under-predict the mass for most of the objects. Typical mass deviations are less than $10 \%$.

Given that dark matter halos assemble via mergers and violent relaxation, it is somewhat surprising that the AC model reproduces the simulation results so well. The success of the model seems to imply that the effect of central baryon condensation on the dark matter distribution is independent of the way in which this condensation is assembled. It may also be due to the fact that the central region is dominated by particles from a single densest progenitor. If the progenitor halo contracts in response to the cooling of baryons early on and then approximately preserves the shape of its inner density profile during subsequent mergers, as suggested by collisionless merger simulations discussed next, the AC model applied to the final mass distribution would work.

\section{How steep is the central dark matter profile?}

Our results have important implications for the efforts to test predictions of the CDM model observationally. The test that received much attention recently is the density distribution in the inner regions of galaxies and clusters. Collisionless simulations indicate that the dark matter density profile in the absence of gas dissipation develops an inner cusp, $\rho(r) \propto r^{-\gamma}$. Using our AC model, we can analytically predict the change of the asymptotic inner slope $\gamma$ due to the condensation of baryons. If the final baryon density at $r / r_{\text {vir }} \ll 1$ can also be written as a power law, $\rho_{\mathrm{b}}(r) \propto r^{-\nu}$, then the post-contraction dark matter density is

$$
\rho(r) \propto r^{-\gamma^{\prime}}, \quad \gamma^{\prime}=\frac{\gamma+(3-\gamma) w \nu}{1+(3-\gamma) w} .
$$

For $\nu=1$, which corresponds to a Hernquist profile or an exponential disk, the asymptotic slope of an NFW profile $(\gamma=1)$ after the contraction remains the same, $\gamma^{\prime}=1$. If the baryons are more concentrated, $\nu>\gamma$, the final slope becomes steeper than the initial slope, $\gamma^{\prime}>\gamma$. For an isothermal sphere, $\nu \approx 2$ (and $w \approx 1$ in our model) the inner slope of the dark matter profile will be close to the slope of the baryon profile (Gnedin et al. 2004).

For realistic cases, the profiles of dark matter and baryons on scales $r \sim 0.01 r_{\text {vir }}$ should be quite similar. These scales are exactly where the mass profiles of massive ellipticals and central cluster galaxies are probed by spectroscopic measurements and gravitational lensing (e.g., Treu \& Koopmans 2004, Sand et al. 2004) and attempts are made to infer the distribution of dark matter in these systems by subtracting the contribution of baryons from the total profile. Since stars dominate the mass in the inner regions, only a small over-estimate of the stellar mass-tolight ratio may lead to a significant under-estimate of the residual dark matter density. Better results can be obtained by using the observed baryon distribution to calculate the contracted dark matter profile and fitting the sum of the two to the total measured mass profile. 
An important hypothesis has been put forth recently by Loeb \& Peebles (2003) and Gao et al. (2004) that the NFW profile is a dynamical attractor, in the sense that remnants of dissipationless mergers are driven to a profile with the central cusp $\rho(r) \propto r^{-1}$, even if their progenitors had steeper profiles. Under this hypothesis, after an early epoch of star formation in elliptical galaxies, subsequent dissipationless mergers would erase the cooling-induced central concentration of dark matter. Together with observation that stars dominate in the centers of galaxies, it implies that the dark matter profile in galaxies which experienced dissipationless mergers is shallower than $r^{-1}$.

Our results do not support the attractor hypothesis. In the cluster runs with cooling and star formation, the growth of the $\mathrm{cD}$ galaxy leads to a significant increase of the dark matter mass enclosed within the inner $10 h^{-1} \mathrm{kpc}$ (four times that in the adiabatic runs). The dark matter density increases, not decreases, at lower redshifts. Even when we assume, as Gao et al., that star formation and gas cooling in massive ellipticals effectively stop at $z \approx 2$ and rerun the simulation with subsequent evolution being purely dissipationless, we still find that the dark matter distribution is steeper than in the adiabatic case (but less so than in the original run where cooling continued until $z=0$ ). Collisionless mergers that the main cluster progenitor has undergone since $z=2$ apparently have not erased the steepening of the profile due to the cooling.

Controlled experiments by Boylan-Kolchin \& Ma (2004) and Kazantzidis et al. (2004) also show that cuspy density profiles are preserved in dissipationless mergers. The merger remnant of two identical halos with cuspy profiles retains the initial inner slope, while mergers of the cored and cuspy halos produce a cuspy remnant. Their analysis shows that the density cusp is remarkably stable and the merger remnants retain the memory of the density profiles of their progenitors. Thus the effects of baryon dissipation in the progenitors are retained, at least partially, in the density distribution of their descendant.

CONTRA is a publicly available code that calculates the contraction of a dark matter halo in response to condensation of baryons in its center. It is available for download at http://www.astronomy.ohio-state.edu/ ognedin/contra/

\section{References}

Blumenthal, G. R., Faber, S. M., Flores, R., \& Primack, J. R. 1986, ApJ, 301, 27

Boylan-Kolchin, M. \& Ma, C. 2004, MNRAS, 349, 1117

Gao, L., Loeb, A., Peebles, P. J. E., White, S. D. M., \& Jenkins, A. 2004, ApJ, 614, 17

Gnedin, O. Y., Kravtsov, A. V., Klypin, A. A., \& Nagai, D. 2004, ApJ, 616, 16

Kazantzidis, S. et al. 2004, ApJ, 611, L73

Kravtsov, A. V. 1999, Ph.D. thesis, New Mexico State University

Loeb, A. \& Peebles, P. J. E. 2003, ApJ, 589, 29

Sand, D. J., Treu, T., Smith, G. P., \& Ellis, R. S. 2004, ApJ, 604, 88

Sellwood, J. A., McGaugh, S. S. 2005, ApJ, in press; astro-ph/0507589

Treu, T. \& Koopmans, L. V. E. 2004, ApJ, 611, 739 